frontal lobes of the non-bypass group who underwent procedures such as EDAS. The reduction of CBF was less after hyperventilation, especially in the frontal lobes of the bypass group in which a much better collateral blood flow occurs.

\title{
POST-HAEMORRHAGIC HYDROCEPHALUS: OUTCOME OF SHUNTING
}

The neuromotor outcome of 33 survivors of grade 3 or 4 neonatal posthaemorrhagic hydrocephalus assessed at a mean age of 4.7 years is reported from the Department of Paediatric Neurology, The Royal Hospital for Sick Children, Edinburgh and the Western General Hospital, Edinburgh. Twothirds of the children were moderately or severely impaired. Raised intracranial pressure was not significantly different between outcome groups. Of 27 shunted, those with 5 or more operations (10 children) had abnormal neurological outcomes. The rise in morbidity after the 4 th shunt was associated with ventriculitis. Outcome was unrelated to the grade of intraventricular hemorrhage or parenchymal changes on ultrasound or CT scanning and was independent of antenatal and perinatal factors. (Lin JP et al. Neurological outcome following neonatal post-haemorrhagic hydrocephalus: the effects of maximum raised intracranial pressure and ventriculo-peritoneal shunting. Child's Nerv Syst June 1992; $\underline{8}: 190-197$. (Correspondence: J.P. Lin, M.D., Department of Paediatric Neurology, The Royal Hospital for Sick Children, Sciennes Road, Edinburgh, EH9 2EP, UK.)

COMMENT. This study shows that a rise in intracranial pressure in the acute stage of hydrocephalus does not carry a significant risk of further handicap or visual impairment. An adverse outcome was statistically related to more than 4 shunt procedures and ventriculitis. The authors' criteria for VP shunting were: 1) increasing ventriculomegaly, 2) raised ICP on monitoring, including during sleep, and 3) abnormal cerebral perfusion as shown by repeated Doppler ultrasonography studies of cerebral blood flow velocity.

The surgical management of post-haemorrhagic hydrocephalus is reported in 22 low birth weight infants from the Department of Neurosurgery, Eastern Virginia Medical School, Norfolk, VA (Gurtner $P$ et al. Child's Nerv Syst June 1992; $\underline{8}: 198-202)$. The use of subcutaneous ventricular reservoirs and a low pressure neonatal shunt was associated with a significant reduction in the morbidity and mortality compared to the use of external ventricular drainage devices.

The use of SPECT for the measurement of cerebral blood flow changes in 4 children with hydrocephalus is reported from the Departments of Neurosurgery and Radiology, Tokai University School of Medicine, Bohsei-dai, Isehara, Kanagawa, Japan (Shinoda M et al. Child's Nerv Syst June $1992 ; \underline{8}: 219-221)$. A positive reabsorption ratio of radioactivity counted in early and delayed images was correlated with a favorable outcome in 3 patients. 
An overview of the surgery of hydrocephalus: past, present and future, by Hirsch JF, Hopital Necker Enfants Malades, Paris, France is published in Acta Neurochir (Wien) 1992; 116:155-160. The need for research in the causes and prevention of hydrocephalus is stressed.

\section{NEONATAL CEREBRAL VENOUS THROMBOSIS}

The clinical presentation and management of 7 neonates with idiopathic cerebral venous thrombosis (CVT) are reported from the Floating Hospital for Infants and Children, New England Medical Center Hospitals, Tufts University School of Medicine, Boston, MA. Unexplained seizures in 3 infants and unexplained lethargy in 4 led to neurological evaluation and the diagnosis of CVT. Follow-up neurological evaluation through 6 months of age showed normal development. Conventional magnetic resonance images indicated more extensive thrombosis than was suggested by cranial CT. MR phase imaging confirmed thrombosis and established absence of blood flow in cerebral veins or sinuses. No infant received treatment with anticoagulants. (Rivkin MJ et al. Neonatal idiopathic cerebral venous thrombosis: an unrecognized cause of transient seizures or lethargy. Ann Neurol July 1992; 32:51-56.) (Correspondence: Dr. Rivkin, New England Medical Center Hospitals, Box 330, Division of Pediatric Neurology, 750 Washington St., Boston, MA 02111.)

COMMENT. Cerebral venous thrombosis may explain transient neonatal seizures or lethargy in newborn infants and may be diagnosed by MR and MR phase imaging.

\section{METABOLIC DISORDERS}

\section{HYDROXYGLUTARIC ACIDEMIA}

Increased urinary excretion of L-2-hydroxyglutaric acid, found in 8 mentally retarded patients from 5 unrelated families including 3 pairs of siblings seen in various centers in Europe, was reported from University Hospital Amsterdam, the Netherlands. The first symptoms occurred between 1 and 5 years of age and consisted of abnormal gait. Febrile seizures occurred in 7 of 8 patients. Cerebellar dysfunction was the principle neurological abnormality in later childhood and adulthood. MRI revealed subcortical leukoencephalopathy, cerebellar atrophy, and changes in the putamina and dentate nuclei. L-2-hydroxyglutaric acid was also increased in the CSF and plasma. Lysine was increased in CSF and plasma and glutaric acid was increased in the plasma of 2 patients. Loading and dietary studies failed to reveal the origin of the L-2-hydroxyglutaric acid. (Barth PG et al. L-2-Hydroxyglutaric acidemia: a novel inherited neurometabolic disease. Ann Neurol July 1992; $\underline{32}: 66-71$.)

COMMENT. Glutaric acidemia, an autosomal recessively inherited disease caused by deficiency of glutaryl-Coenzyme A dehydrogenase, was manifested by acute dystonia in 3 infants reported from the Children's Hospital of Pittsburgh, PA (See Ped Neurol Briefs Jan 